\title{
Multiobjective TOU Pricing Optimization Based on NSGA2
}

\author{
Huilan Jiang, Bingqi Liu, Yawei Wang, and Shuangqi Zheng \\ Key Laboratory of Smart Grid of Ministry of Education, Tianjin University, Tianjin 300072, China \\ Correspondence should be addressed to Huilan Jiang; hljiang@tju.edu.cn
}

Received 24 January 2014; Accepted 27 June 2014; Published 13 July 2014

Academic Editor: Gongnan Xie

Copyright (C) 2014 Huilan Jiang et al. This is an open access article distributed under the Creative Commons Attribution License, which permits unrestricted use, distribution, and reproduction in any medium, provided the original work is properly cited.

\begin{abstract}
Fast and elitist nondominated sorting generic algorithm (NSGA2) is an improved multiobjective genetic algorithm with good convergence and robustness. The Pareto optimal solution set using NSGA2 has the character of uniform distribution. This paper builds a time-of-use (TOU) pricing mathematical model considering actual constraint conditions and puts forward a new method which realizes multiobjective TOU pricing optimization using NSGA2. A variety of objective TOU pricing schemes can be provided for decision makers compared with traditional method. Furthermore, the multiple attribute decision making theory is applied in processing the Pareto optimal solution set to calculate the optimal compromise price scheme. The simulation results have shown that the TOU pricing scheme determined by the method proposed above can achieve a better effect of clipping the peak load to fill the valley load. Consequently, the study in this paper is innovative and is a successful exploration of coordinating the relation of various objective functions concerned in TOU pricing optimization problem.
\end{abstract}

\section{Introduction}

Time-of-use price is an important demand-side management (DSM) method, which could stimulate and encourage the consumers to change the consumption behaviors and achieve the purpose of clipping the peak load to fill the valley load as well as changing the load curve, so that the economy, security, and reliability of the power system are improved. So far, three kinds of TOU pricing are studied: the first is based on the relation between sale and retail prices [1], the second is based on the consumer response curve or price elasticity matrix $[2,3]$, and the third is based on game control [4]. However, the research on the optimization methods, which can decrease peak load and peak-valley difference at the same time, has not been studied enough. It is a typical multivariable constrained multiobjective optimization problem.

The traditional algorithms of multiobjective TOU pricing optimization are weighted method [5], constraint method [6], and so on. The multiobjective optimization problem is transferred into a single objective optimization problem. Then, the problem is solved by the relatively mature single objective optimization algorithm in which only one solution is obtained. However, there is a solution set in the multiobjective optimization problem. Moreover, the weights of the objectives are defined subjectively rather than objectively. So, the optimal extent is affected. Multiobjective genetic algorithm is an evolutionary algorithm. Its core thought is to coordinate the relation between the objective functions to search for optimal solution set [7]. NSGA2, an improved multiobjective generic algorithm, overcomes the shortcomings mentioned above and has the characters of good convergence and excellent robustness. Its noninferior solutions are uniformly distributed in objective space. Considering TOU pricing is a multivariable constrained multiobjective optimization problem; this paper uses NSGA2 to solve the problem and puts forward a new kind of TOU pricing optimization mechanism which can achieve a better effect in balancing the relation between the objective functions. Case analysis shows that the proposed TOU pricing multiobjective optimization based on NSAG2 takes on better effectiveness and superiority.

\section{Multiobjective Optimization Pareto Optimal Solution Set and Pareto Genetic Algorithm}

2.1. The Concept of Nondominated Solution (Pareto Solution). Given two solutions, $\mathbf{X}_{u}$ and $\mathbf{X}_{v}$, if all the objectives of $\mathbf{X}_{u}$ are 
superior to the objectives of $\mathbf{X}_{v}$, we say that $\mathbf{X}_{u}$ dominates $\mathbf{X}_{v}$ or $\mathbf{X}_{v}$ is dominated by $\mathbf{X}_{u}$. If $\mathbf{X}_{u}$ is not dominated by the others, it is called nondominated solution or Pareto solution. The set of nondominated solutions is called Pareto front. The solution of Pareto front is not dominated by the ones outside the Pareto front. Compared with other solutions, Pareto solution has less objective conflicts; thus, a better selection space is supplied for the decision maker.

2.2. The Advantages of Pareto Front and NSGA2. For a multiobjective optimization problem, the optimal solution is not unique, and it is a set of many satisfied solutions, which is called Pareto optimal set. Pareto optimal set can provide more than one optional solution for decision makers. According to the practical needs and combined with the theory of multiple attribute decision making, decision maker can choose an optimal compromise solution [8], which owns the highest satisfaction.

In the multiobjective optimization, there may be conflicts between different objectives and it is hard to make a judicious decision by comparing all these objectives. If a solution is the best one based on a certain objective, it may be weakly worse for the other objectives. The core of multiobjective genetic algorithms is to coordinate the relation between the objective functions. Nondomination rank and selection operator are used to make the population evolve towards Pareto optimal set in the formulation of the problem. Then, the Pareto optimal set is obtained, which can make the objective function relatively maximized (or relatively minimized). Thus, several kinds of multiobjective genetic algorithms based on Pareto optimal set come into being, in which nondominated sorting generic algorithm (NSGA) [9] is a typical example. NSGA2 [10] is the improvement of NSGA which is proposed by Deb and others. Its prominent characteristics can be listed as follows:

(1) NSGA2 is based on nondomination rank, which makes the complexity decrease from $O\left(K N^{3}\right)$ of NSGA to $O\left(K N^{2}\right)$;

(2) crowding distance comparison operator is adopted for optimization. So that individuals in the population can extend to the entire Pareto domain and maintain the diversity of the population to avoid local convergence;

(3) elitist strategy is used in NSGA2. The next generation is got from the sorting and competition of parent generation and offspring generation. Thus, the quality of the next generation is guaranteed.

\section{The Mathematic Multiobjective Optimization Model}

According to the typical daily load curve, peak, flat, and valley are defined as $T_{p}, T_{f}$, and $T_{v}$, respectively, and their corresponding prices are $P_{p}, P_{f}$, and $P_{v}$. Consumer response curve is $y=f(x)$. The average price or market clearing price before the implementation of TOU pricing is $P_{0}$ which is assumed as a known constant.
3.1. The Selection of Multiobjective Functions. In this paper, minimizing the maximum load and minimizing the peakvalley difference are considered simultaneously as TOU pricing objective functions.

Objective function 1 is as follows: minimizing the maximum load

$$
\min \left(Q_{\max }\right)
$$

Objective function 2 is as follows: minimizing the peakvalley difference

$$
\min \left(Q_{\max }-Q_{\min }\right),
$$

where $Q_{\max }=\max \left[Q\left(P_{f}, P_{p}, P_{g}, T_{f}, T_{p}, T_{g}\right)\right]$ is the maximum of the peak load of the day, $Q_{\min }=\min \left[Q\left(P_{f}, P_{p}, P_{g}\right.\right.$, $\left.\left.T_{f}, T_{p}, T_{g}\right)\right]$ is the minimum of valley loads, $P_{p}, P_{f}$, and $P_{v}$ are the time price of peak, flat, and valley loads, $T_{p}, T_{f}$, and $T_{v}$ are the time period of peak, flat, and valley loads, and $Q\left(P_{p}, P_{f}, P_{v}, T_{p}, T_{f}, T_{v}\right)$ is the new load after the implement of TOU pricing according to the consumer response curve.

3.2. Constraint Conditions. The sum of electricity consumption keeps invariant after the implementation of TOU pricing:

$$
Q_{p}^{\prime}+Q_{f}^{\prime}+Q_{v}^{\prime}=Q_{p}+Q_{f}+Q_{v}
$$

where $Q_{p}, Q_{f}$, and $Q_{v}$ are the peak, flat, and valley loads before the implementation of TOU pricing and $Q_{p}^{\prime}, Q_{f}^{\prime}$, and $Q_{v}^{\prime}$ are the peak, flat, and valley loads after the implementation of TOU pricing.

Considering the production cost and the consumers' endurance, the prices of peak, flat, and valley loads are limited by the price supervision department. The limitations are as follows:

$$
\begin{aligned}
& P_{p \text { min }} \leq P_{p} \leq P_{p \text { max }}, \\
& P_{f \text { min }} \leq P_{f} \leq P_{f \text { max }}, \\
& P_{v \text { min }} \leq P_{v} \leq P_{v \text { max }},
\end{aligned}
$$

where $P_{p \text { min }}, P_{p \text { max }}, P_{f \text { min }}, P_{f \text { max }}, P_{v \text { min }}$, and $P_{v \text { max }}$ are the upper and lower limit prices of peak, flat, and valley loads.

The price of peak, flat, and valley loads varies within limits, and the effect of peak load shifting varies at the same time. This paper mainly studies the optimization of the effect of peak load shifting. $P_{p}, P_{f}$, and $P_{v}$ are obtained according to the optimization of the mathematical model. They are substituted into the following inequality:

$$
(1-\delta) M_{0}<Q_{p}^{\prime} P_{p}+Q_{f}^{\prime} P_{f}+Q_{v}^{\prime} P_{v}<M_{0}
$$

where $\delta=M^{\prime} / M_{0}$ is interest concessions coefficient, $M_{0}$ is the total electricity cost before the implementation of TOU pricing, and $M^{\prime}$ is the reduced electric power cost of the power company after the implementation of TOU pricing. The inequality is used to verify the model and $\delta$ is obtained through the inequality, whose upper limitation is $10 \%$. It is a reference for decision makers to optimize the economic indicator. 


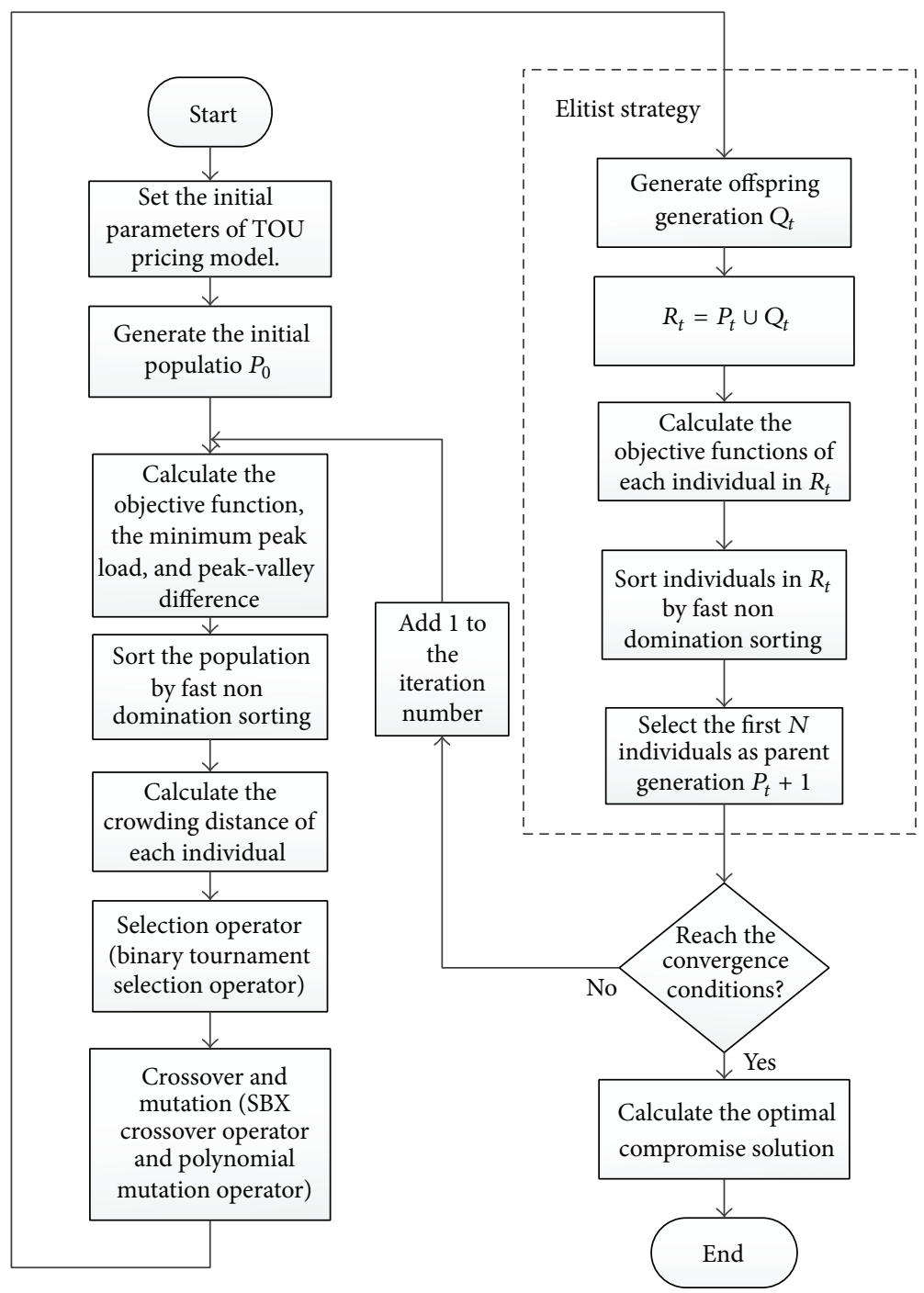

FIGURE 1: Flow chart of optimizing time-of-use price based on NSGA2.

\section{Multiobjective TOU Pricing Optimization Based on NSGA2}

4.1. The Flow of Multiobjective TOU Pricing Optimization Based on NSGA2. Power system TOU pricing is a multiobjective optimization problem. It contains a plurality of objective functions, and objectives have the same decision variables, which interact with each other. It is hard to objectively evaluate the solution got from a single objective optimization or a single objective optimization merged by multiobjective optimization. NSGA2 is used in the multiobjective TOU pricing optimization in this paper, which aims at obtaining the solution of high synthetical satisfaction degree when taking the multiobjective into consideration.

The flow of multiobjective TOU pricing optimization based on NSGA2 is shown in Figure 1.

4.2. Coding. $P_{0}$ is used as the reference. $P_{p}, P_{f}$, and $P_{v}$ are calculated into per unit value $x_{p}, x_{f}$, and $x_{v}$, which are used as control variables. The coding format in real-coded pattern is $\left[x_{f}, x_{p}, x_{v}\right]$. The real decoding value of peak, flat, and valley price is as follows:

$$
\begin{aligned}
& P_{p}=x_{p} P_{0}, \\
& P_{f}=x_{f} P_{0}, \\
& P_{v}=x_{v} P_{0} .
\end{aligned}
$$

4.3. Generate the Initial Population. When generating a random initial population of individuals, the constraint conditions of price are used to limit the random number, to ensure that the random number is generated within the constraints. Then, set the appropriate number of initial population of individuals. It cannot be too small to converge or too big to prolong search time. It also should be selected randomly to ensure the diversity and the convergence to the optimal solution. 
4.4. Fast Nondomination Sorting. Before the process of selection operator for individual populations, according to the level of individual nondomination solutions, the population is sorted by fast nondomination sorting of NSGA2. The specific methods are as follows.

In the algorithm, two parameters, $n_{i}$ and $S_{i}$, of individual $i$ should be calculated. $n_{i}$ is the number of individuals that dominate individual $i . S_{i}$ is the set of individuals that are dominated by individual $i$. The specific steps of fast nondomination sorting are as follows.

(1) Find all the individuals whose parameter $n_{i}$ is 0 . The individuals are preserved in the set $H$, which is the first nondomination layer.

(2) For each individual $i$ of set $H$, traverse each individual $l$ in the set $S_{i}$ and execute $n_{l}=n_{l}-1$. If $n_{l}=0$, preserve individual $l$ in the next nondomination layer.

(3) Repeat the previous step until the entire population is sorted.

For a population, whose number of objective functions is $K(K>1)$ and size is $N$, two parameters, $n_{i}$ and $S_{i}$, are calculated for each individual by NSGA2. Traversing the entire population, the total computational complexity is $O\left(K N^{2}\right)$. Nondomination sorting is used in NSGA, and its computational complexity is $O\left(K N^{3}\right)$. So, the calculation efficiency of fast nondomination is higher.

4.5. Virtual Fitness. In NSGA2, the individual fitness includes nondomination rank and virtual fitness. Crowding distance is used by NSGA2 to form individual fitness to maintain the diversity of the individual and prevent the accumulation of the individual in the local. It is defined as the sum distance of the two points on either side of this point along each of the objectives. In Figure 2, the crowding distance of the $i$ th solution in its front (marked with solid circles) is the sum of sidelength of the cuboid (shown with a dashed box), which is $\left[f_{1}(i+1)-f_{1}(i-1)\right]+\left[f_{2}(i-1)-f_{2}(i+1)\right]$. This approach makes the selected individuals distribute evenly in the objective space with good robustness.

4.6. Selection Operator. Genetic selector operator is for optimization towards Pareto optimal solution set and to make the solutions distribute evenly. The best individual with the greatest probability of survival is preserved by selection operator to avoid the loss of good genes. Thereby, global convergence and computational efficiency are improved. Each individual has two parameters, $i_{\text {rank }}$ (nondomination rank) and $i_{d}$ (crowding distance). Binary tournament selection operator is used in this paper. Two individuals are chosen randomly for comparison. If $i_{\text {rank }}<j_{\text {rank }}$ or $\left(i_{\text {rank }}=j_{\text {rank }}\right.$ and $i_{d}>j_{d}$ ), individual $i$ is better than individual $j$. That is to say, if two individuals are in different layers, the individual with the lowest nondomination rank is selected, which is firstsorted in the process of nondomination sorting with high level of nondomination. Or if two individuals are in the same layer, the individual with the largest crowding distance is selected, which has advantages in selection.

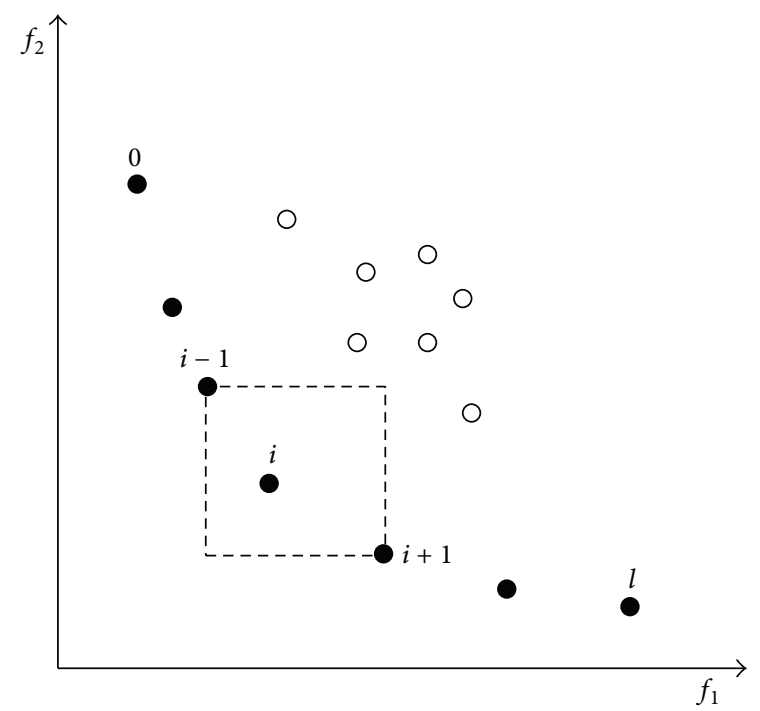

FIGURE 2: Chart of local crowding distance.

4.7. Crossover and Mutation. The cooperation of crossover and mutation makes genetic algorithm perform well in local and global search. SBX crossover operator and polynomial mutation operator are adopted. SBX operator simulates binary crossover process for real-coded parent individuals. For a given random cross-point, switch two sides of the crosspoint of the parent of the individual parts. Polynomial mutation operator is based on polynomial distribution. Offspring generation $Q_{t}$ is generated through crossover and mutation operator.

4.8. Elitist Strategy. Elitist strategy makes excellent individuals retain to the offspring generation directly. It is a necessary condition for the genetic algorithm converging with probability 1 . The method is as follows.

(1) Parent generation $P_{t}$ and offspring generation $Q_{t}$ are combined into a population $R_{t}=P_{t} \cup Q_{t}$.

(2) Sort $R_{t}$ by fast nondomination sorting and calculate crowding distance of every individual. Select individuals one by one based on nondomination rank, until the number of individuals reaches $N$. Then, the new parent generation $P_{t+1}$ is generated.

Iteration is done again and again until convergence is reached. Then, fast nondomination sorting is used to obtain the Pareto optimal solution set, that is, Pareto front, which consists of the individuals in the first nondomination layer.

4.9. Optimal Compromise Solution. The optimal compromise solution is the solution in the Pareto front with the greatest satisfaction. 
In Pareto optimal solution set, the satisfaction of each objective function of each Pareto solution is represented by fuzzy membership function as follows:

$$
h_{i}= \begin{cases}1, & f_{i} \leq f_{i \min }, \\ \frac{f_{i \max }-f_{i}}{f_{i \max }-f_{i \min }}, & f_{i \min }<f_{i}<f_{i \max }, \\ 0, & f_{i} \geq f_{i \max },\end{cases}
$$

where $f_{i}$ is the objective function and $i=1,2, \ldots, N_{\mathrm{obj}} . N_{\mathrm{obj}}$ is the number of object functions. $f_{i \min }$ and $f_{i \max }$ are the maximum and minimum of the $i$ th objective function.

According to formula (8), satisfaction of each Pareto solution is calculated. Then, the solution with the greatest $h$ value is chosen as the optimal compromise solution

$$
h=\sum_{i=1}^{N_{\text {obj }}} h_{i}
$$

\section{Case and Analysis}

In order to verify the effectiveness and feasibility of NSGA2 in multiobjective TOU pricing optimization, large industrial consumers in a specific region are chosen to be optimized by NSGA2. The result is analyzed subsequently.

\subsection{The Data before TOU Pricing Optimization}

(1) Typical Daily Load Data. The typical daily load data is shown in Table 1.

(2) The Price and the Cost of Power Consumers. In the region, the average price or market clearing price is $P_{0}=$ $¥ 0.5647 / \mathrm{kW} \cdot \mathrm{h}$. The cost of large industrial power consumers is $M_{0}=¥ 8.80 \times 10^{6}$.

\section{(3) Periods Division and Consumer Response Curve}

Peak period: 08:00 12:00 (Peak 1), 18:00 22:00 (Peak 2);

Flat period: 12:00 18:00 (Flat 1), 22:00 24:00 (Flat 2);

Valley period: 00:00 04:00 (Valley 1), 04:00 08:00 (Valley 2).

Through investigation, consumer response curve is obtained, whose expression is $y=f(x)=-0.2807 x+1.2952$, where $x$ is the per unit value of price and $y$ is the per unit load value of consumer.

(4) Constraint Conditions of Peak, Flat, and Valley Price. In the region, according to the power cost and consumers' endurance, the constraint conditions of peak, flat, and valley price are regulated as follows (shown in per unit value): $0.3 \leq$ $x_{v} \leq 0.8,0.8 \leq x_{f} \leq 1.2,1.2 \leq x_{p} \leq 1.8$.

5.2. The TOU Pricing Optimization by NSGA2. In the calculation case, the parameters settings of NSGA2 are as follows: the
TABLE 1: Data of the large industrial load.

\begin{tabular}{lc}
\hline Time (hour of the day) & Load (MW) \\
\hline 1 & 511.0 \\
2 & 532.5 \\
3 & 520.0 \\
4 & 491.0 \\
5 & 543.5 \\
6 & 566.0 \\
7 & 596.0 \\
8 & 643.5 \\
9 & 717.5 \\
10 & 734.0 \\
11 & 752.5 \\
12 & 670.0 \\
13 & 682.5 \\
14 & 692.5 \\
15 & 686.0 \\
16 & 686.0 \\
17 & 729.0 \\
18 & 735.0 \\
19 & 778.0 \\
20 & 775.0 \\
21 & 729.0 \\
22 & 681.0 \\
24 & 581.5 \\
\hline
\end{tabular}

population size is 500 ; the iterative time is 500 ; the crossover rate is 0.9 ; the mutation rate is 0.1 .

In NSGA2, the selection basis of individuals is the indexes of nondomination rank and crowding distance. For the individuals in parent generation, objective functions are calculated by the mathematic multiobjective optimization model. Then, 250 individuals $(N / 2=250)$ are selected according to nondomination rank and crowding distance. They are used to crossover and mutate to generate the offspring generation. The parent generation and the offspring generation are combined together to generate the new parent generation by elitist strategy.

The case takes the 10th generation for example. Table 2 shows the individuals in the first 3 layers sorted by fast nondomination sorting. The distribution of these individuals in the objective space is shown in Figure 3. The crowding distance of the individuals in the Pareto front is shown in Table 3. From Figure 3, it is known that the accumulation degree of each nondomination layer is different, so that the global optimization is ensured. Crowding distance is taken into consideration when selecting individuals to ensure the diversity and avoid the local convergence.

When the convergent conditions are met, the iteration goes to an end. Then, the final Pareto optimal solution set (Pareto front) is obtained. A part of the representative Pareto optimal solutions is shown in Table 4. The distribution of Pareto front and the optimal compromise solution are shown in Figure 4 . The optimal compromise price scheme 
TABLE 2: The first 3 layers of the 10th generation (accurate to 0.001).

\begin{tabular}{lccc}
\hline Layer & Chromosome coding & Max peak load (MW) & Max peak-valley difference (MW) \\
\hline & {$[1.1246,1.3656,0.4762]$} & 710.5655 & 146.3994 \\
1st & {$[1.1215,1.3333,0.4853]$} & 707.1186 & 146.7428 \\
& {$[1.1207,1.3335,0.4826]$} & 708.7085 & 146.5791 \\
& {$[1.1417,1.3392,0.4197]$} & 704.6405 & 147.4979 \\
\hline & {$[1.1392,1.3344,0.4168]$} & 704.4741 & 147.5799 \\
\hline \multirow{3}{*}{ 2nd } & {$[1.1418,1.3403,0.4182]$} & 704.9236 & 147.8027 \\
& {$[1.0976,1.3339,0.3931]$} & 707.7580 & 147.0330 \\
& {$[1.1206,1.3335,0.4828]$} & 709.1306 & 146.5835 \\
& {$[1.1239,1.3655,0.4767]$} & 710.6913 & 146.4315 \\
& {$[1.1450,1.3352,0.4197]$} & 704.5676 & 148.0924 \\
3rd & {$[1.0999,1.3206,0.4293]$} & 708.4119 & 146.9282 \\
& {$[1.1448,1.3378,0.4200]$} & 704.7100 & 148.0073 \\
& {$[1.1438,1.3336,0.4181]$} & 704.5684 & 148.1748 \\
& {$[1.1224,1.3648,0.4761]$} & 710.8333 & 146.4984 \\
& {$[1.1193,1.3371,0.4772]$} & 709.2855 & 146.6396 \\
& {$[1.1447,1.3344,0.4199]$} & 704.5933 & 148.1420 \\
& {$[1.1204,1.3483,0.4813]$} & 710.1927 & 146.5897 \\
& {$[1.1421,1.3412,0.4183]$} & 704.9940 & 147.8267 \\
\hline
\end{tabular}

TABle 3: The crowding distance of the individuals in Pareto front.

\begin{tabular}{lccc}
\hline Number & $\begin{array}{c}\text { Peak load } \\
(\mathrm{MW})\end{array}$ & $\begin{array}{c}\text { Peak-valley } \\
\text { difference (MW) }\end{array}$ & Crowding distance \\
\hline 1 & 710.5655 & 146.3994 & Boundary point \\
2 & 707.1186 & 146.7428 & 2.0367 \\
3 & 708.7085 & 146.5791 & 3.2332 \\
4 & 704.6405 & 147.4979 & 5.2352 \\
5 & 704.4741 & 147.5799 & Boundary point \\
\hline
\end{tabular}

(the third solution in Table 4) is that the peak price is $¥ 0.7677 / \mathrm{kWh}$, the flat price is $¥ 0.6541 / \mathrm{kWh}$, and the valley price is $¥ 0.2542 / \mathrm{kWh}$. The corresponding max peak load is 703.4001 MW, and the peak-valley difference is $144.8588 \mathrm{MW}$. And the original max peak load is $778.0 \mathrm{MW}$, and the peakvalley difference is $287.0 \mathrm{MW}$. By contrast, the optimization by NSGA2 has great effects on clipping the peak load to fill the valley load.

5.3. Analysis and Comparison. (1) More than one objective solution is obtained by NSGA2, but only one is obtained by traditional algorithm.

It is seen in Figure 4 that the two objectives, the minimum peak load and the minimum peak-valley difference, have a contradiction. It is impossible to find a solution which is optimal for two objectives at the same time. Therefore, decision makers can make choices by objective needs in Pareto optimal solutions. If the minimum peak load is used as the main objective, the solution is selected from the upper left part of the Pareto solution set, such as the first solution in Table 4. If the minimum peak-valley difference is used as the main objective, the solution is selected from the

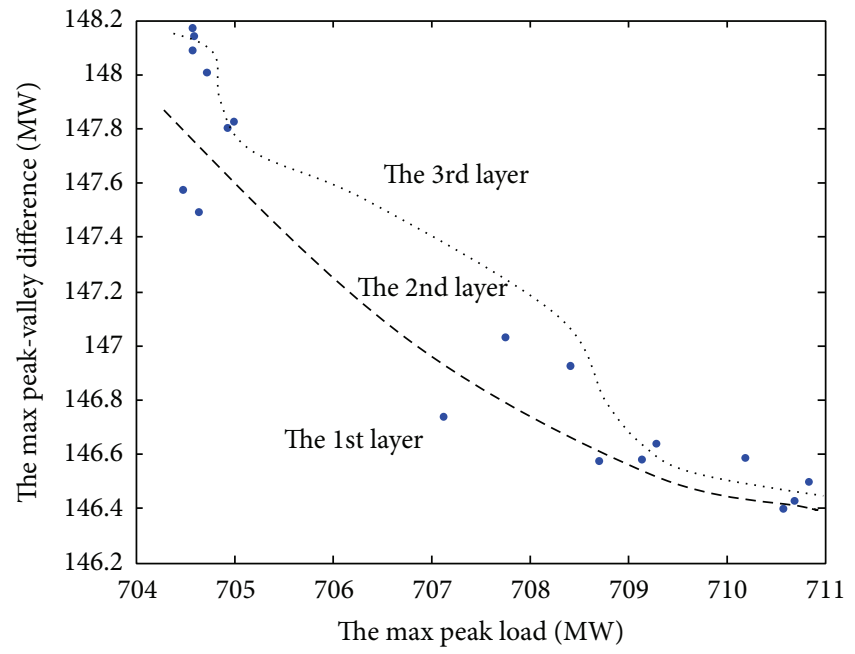

FIgURE 3: The distribution of individuals in the first three layers in objective space.

lower right part of the Pareto solution set, such as the fifth solution in Table 4. If the preference objective function is not specified, the optimal compromise solution is selected, just at the marked point in Figure 4. The load curve of optimal compromise solution is shown in Figure 5. Multiobjective optimization is transferred into single objective in traditional algorithm. However, the weights of each objective function are artificially regulated. The blindness and subjectivity of traditional optimization algorithm are avoided by NSGA2, thus realizing multiobjective TOU pricing optimization.

(2) Comparison with Traditional Method. The weighted method is a traditional single objective optimization method. 
TABle 4: A set of Pareto optimal solutions.

\begin{tabular}{|c|c|c|c|c|c|c|}
\hline Number & Flat price $(\mathrm{pu})$ & Peak price $(\mathrm{pu})$ & Valley price $(\mathrm{pu})$ & Max peak load (MW) & Peak-valley difference (MW) & $h$ value \\
\hline 1 & 1.1582 & 1.3581 & 0.4501 & 703.3022 & 144.8618 & 0.9817 \\
\hline 2 & 1.1583 & 1.3586 & 0.4502 & 703.3366 & 144.8599 & 1.0475 \\
\hline 3 & 1.1583 & 1.3595 & 0.4502 & 703.4001 & 144.8588 & 1.3222 \\
\hline 4 & 1.1584 & 1.3599 & 0.4504 & 703.4206 & 144.8531 & 0.9930 \\
\hline 5 & 1.1585 & 1.3606 & 0.4505 & 703.4647 & 144.8493 & 0.9567 \\
\hline
\end{tabular}

TABLE 5: Contrast of NSGA2 and weighted method.

\begin{tabular}{lllcr}
\hline Method & \multicolumn{2}{c}{ Case } & Max peak load (MW) & Peak-valley difference (MW) \\
\hline NSGA2 & \multicolumn{2}{c}{} & 703.4001 & 144.8588 \\
\hline & $w_{1}=0.1$ & $w_{2}=0.9$ & 704.8171 & 144.3526 \\
Weighted method & $w_{1}=0.3$ & $w_{2}=0.7$ & 703.6927 & 144.7283 \\
& $w_{1}=0.5$ & $w_{2}=0.5$ & 703.4111 & 145.1413 \\
& $w_{1}=0.7$ & $w_{2}=0.3$ & 703.3120 & 145.8738 \\
& $w_{1}=0.9$ & $w_{2}=0.1$ & 703.2972 & 146.0622 \\
\hline
\end{tabular}

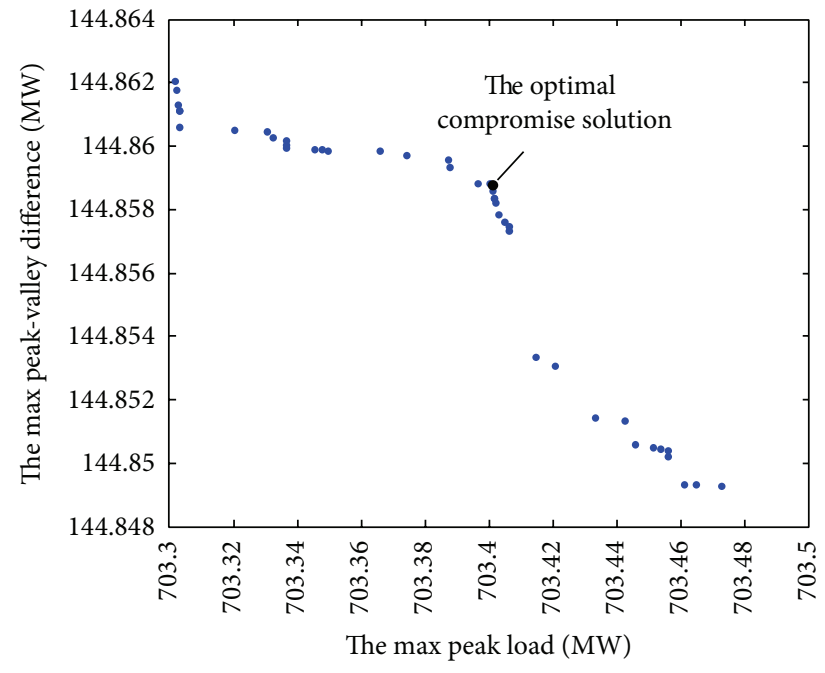

Figure 4: Pareto front.

When used for solving the multiobjectives optimization problem, the weighted method would transfer the problem into a single objective optimization problem by assigning a set of weight value to each objective. In this paper, The weighted method is also used in optimizing TOU pricing problem considering setting different weight values $\left(w_{1}\right.$ and $\left.w_{2}\right) . w_{1}$ is the weight value of the objective function 1 , the maximum load. $w_{2}$ is the weight value of the objective function 2, the peak-valley difference. Besides, $w_{1}+w_{2}=1$. Compared with NSGA2, the simulation results of these two methods are shown in Table 5.

It can be seen in Table 5 that different weight value distribution in the weighted method would lead to different solutions. Generally, the bigger the weight value of an objective is, the better the solution of corresponding objective is, since this objective is emphasized. When an objective is

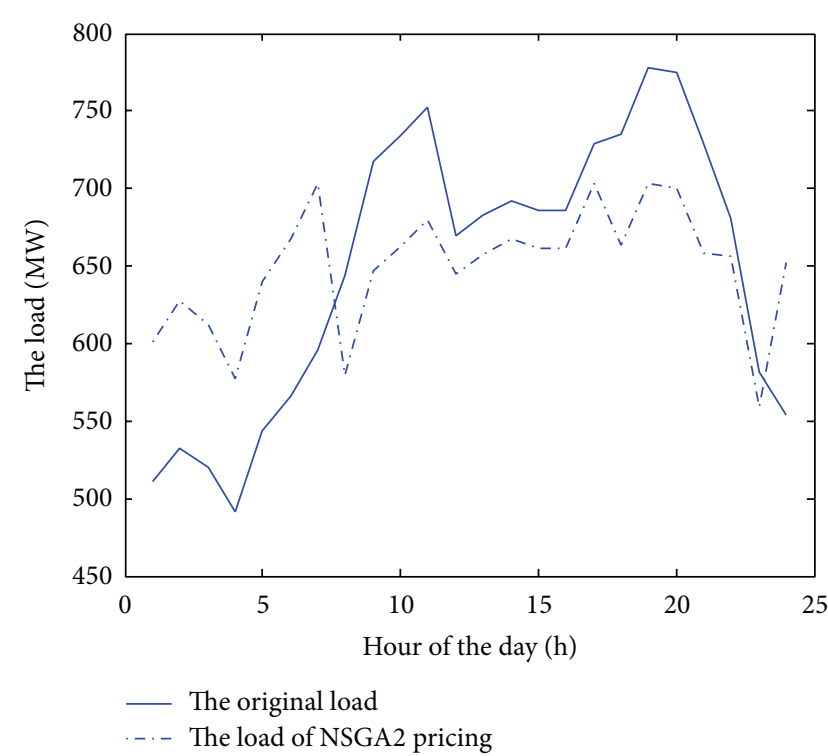

FIGURE 5: Contrast of load curve between optimization by NSGA2 and the original one.

overemphasized, the corresponding objective function value is superior to that of NSGA2, while the other objective function values would be much worse, just as the case of $w_{1}=0.1 w_{2}=0.9$ shown in Table 5. When the weight values are chosen improperly, all the objective function values are worse than those of NSGA2, just as the case of $w_{1}=0.5 w_{2}=0.5$ shows. For the weighted method, it is important but difficult to choose suitable weight of objectives because the weights are determined subjectively. By contrast, the optimal compromise solution of NSGA2 is obtained objectively. In conclusion, the TOU pricing scheme of NSGA2 has a significant advantage on clipping the peak load to fill the valley load. 


\section{Conclusions}

NSGA2 is a newly developed algorithm. For multiobjective TOU pricing optimization, a new thought is put forward in this paper. NSGA2 is applied in TOU pricing, and more than one Pareto solutions are obtained. Choices are supplied for the decision makers for selection, which overcomes the shortcomings of the traditional algorithm and realizes the multiobjective TOU pricing optimization. Example analysis demonstrates that the remarkable effect of clipping the peak load to fill the valley load is achieved by NSGA2. Good technique support is supplied for power market operation which is affected by the industrial and economic structure.

\section{Conflict of Interests}

The authors declare that there is no conflict of interests regarding the publication of this paper.

\section{References}

[1] D. W. Duan, J. Y. Liu, and J. G. Wu, "Optimal TOU retail pricing models for distribution utility with risk management," Automation of Electric Power Systems, vol. 24, no. 2, pp. 8-12, 2005.

[2] Z. Qin, S. Yue, Y. Yu, W. Chen, S. Wang, and X. Pu, "Price elasticity matrix of demand in current retail power market," Automation of Electric Power Systems, vol. 28, no. 5, pp. 16-24, 2004.

[3] G. Q. Liu, J. Zhang, and H. Liu, "Research on TOU pricing based on consumer response curve," Journal of North China Electric Power University, vol. 32, no. 3, pp. 25-27, 2005.

[4] Y. L. Ren, J. Li, and Z. Yang M, "research on time-of-use power price based on game control," Science and Technology Management Research, no. 2, pp. 180-183, 2006.

[5] W. Ding, J. Yuan, and Z. Hu, "Time-of-use price decision model considering users reaction and satisfaction index," Automation of Electric Power Systems, vol. 29, no. 20, pp. 10-14, 2005.

[6] Z. F. Tan, M. B. Wang, and J. X. Qi, “TOU pricing model and fuzzy optimization method for solving," Systems EngineeringTheory \& Practice, vol. 28, no. 9, pp. 145-151, 2008.

[7] E. Zitzler, K. Deb, and L. Thiele, "Comparison of multiobjective evolutionary algorithms: empirical results," Evolutionary Computation, vol. 8, no. 2, pp. 173-195, 2000.

[8] M. Farina and P. Amato, "A fuzzy definition of "optimality" for many-criteria optimization problems," IEEE Transactions on Systems, Man, and Cybernetics A: Systems and Humans., vol. 34, no. 3, pp. 315-326, 2004.

[9] N. Srinivas and K. Deb, "Multi-objective optimal path planning using elitist non-dominated sorting genetic algorithms," Evolutionary Computation, vol. 2, no. 3, pp. 221-248, 1994.

[10] K. Deb, S. Agrawal, and A. Pratap, "A fast elitist non-dominated sorting algorithm for multi-objective optimization: NSGA-II," in Proceedings of the 6th Parallel Problem Solving from Nature Confernece, pp. 182-197, Paris, France, 2002. 


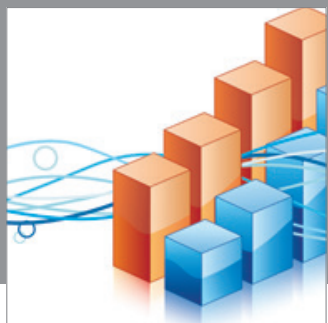

Advances in

Operations Research

mansans

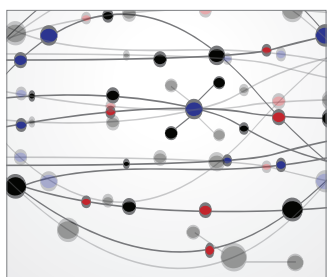

The Scientific World Journal
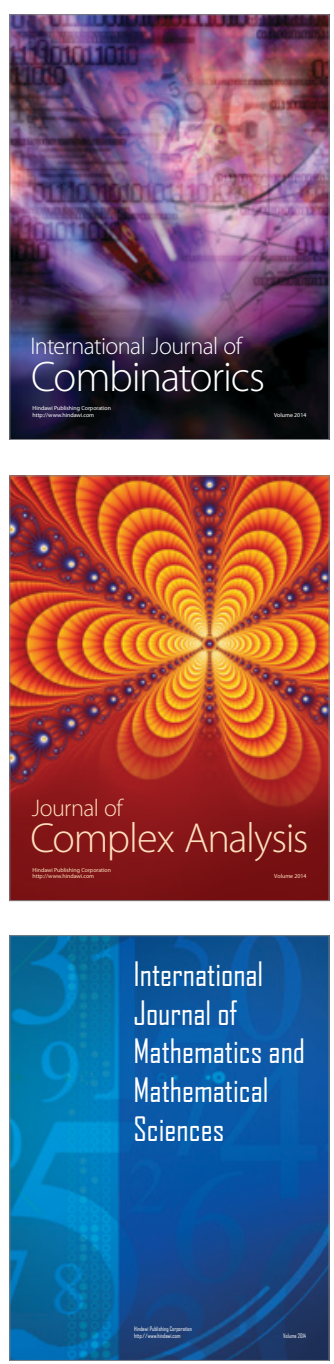
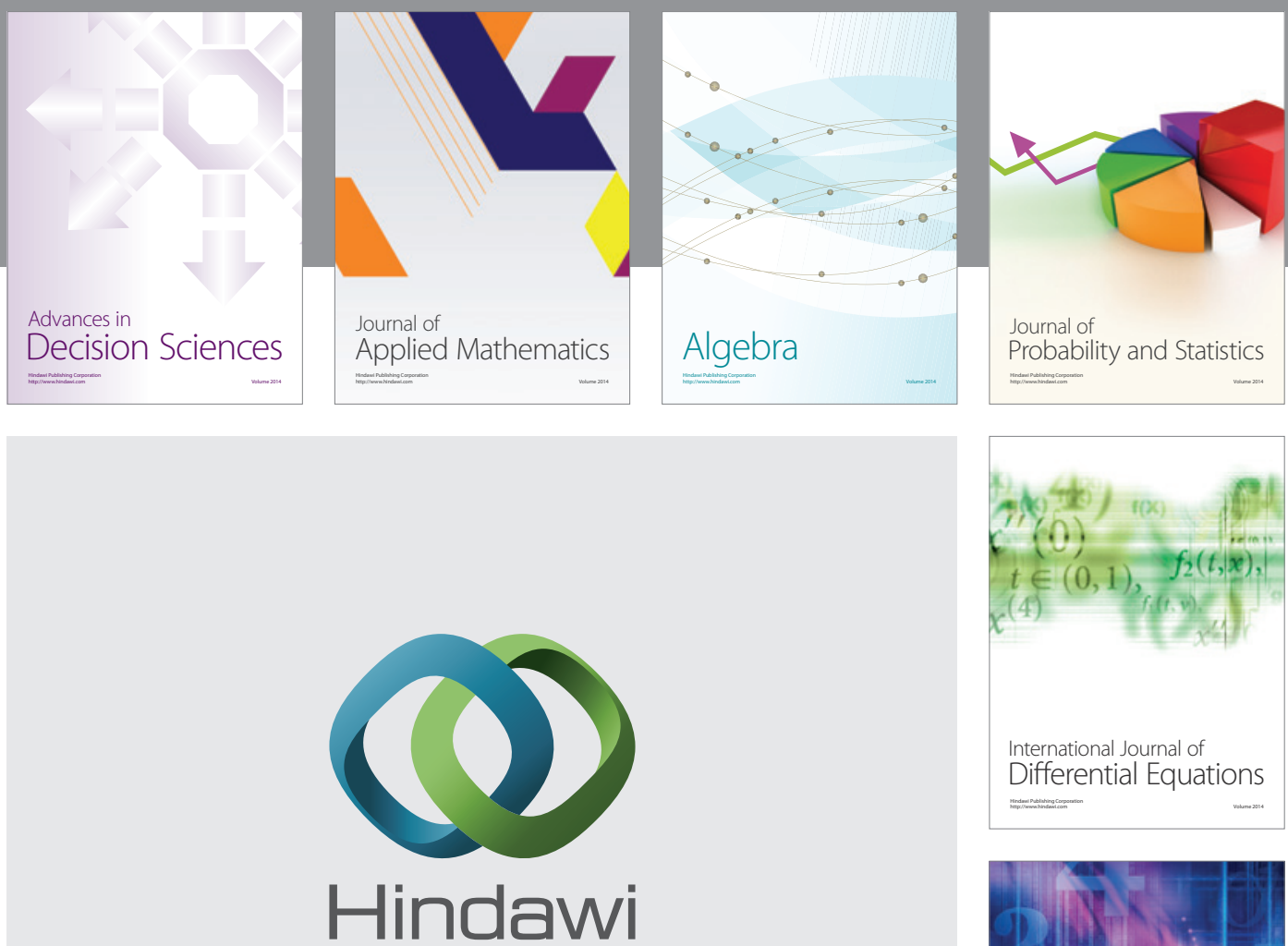

Submit your manuscripts at http://www.hindawi.com
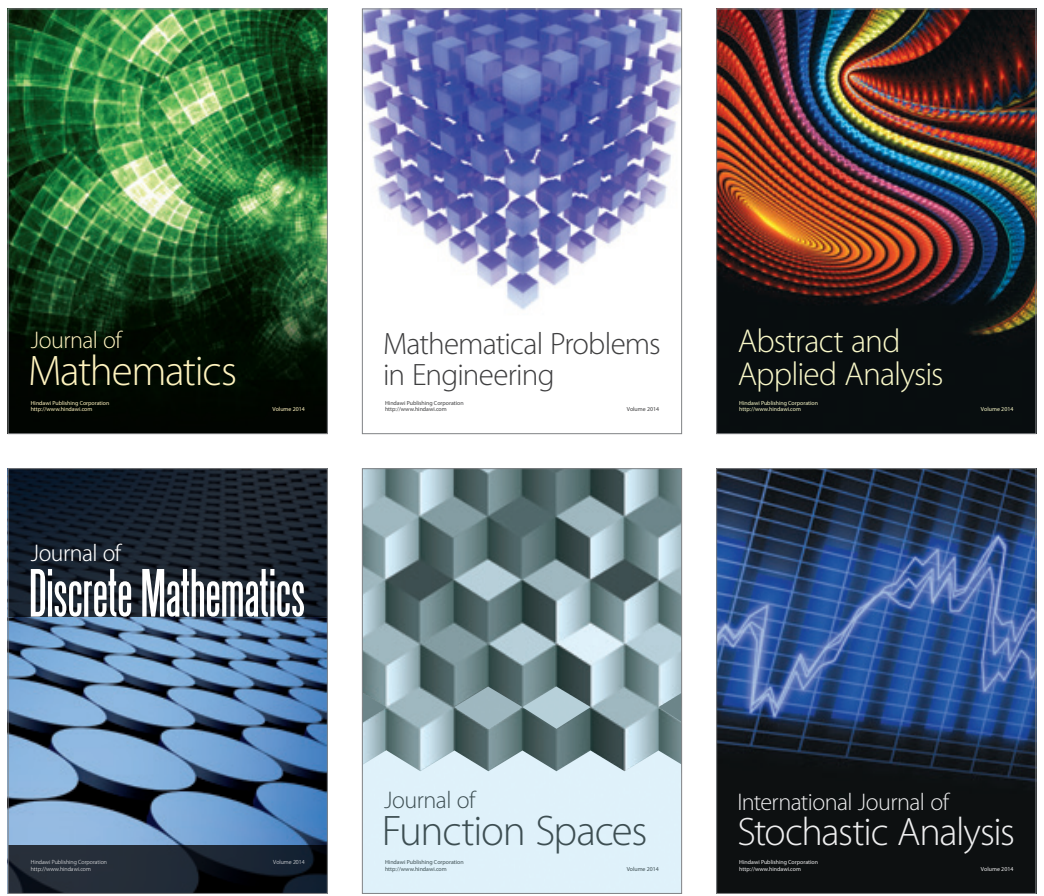

Journal of

Function Spaces

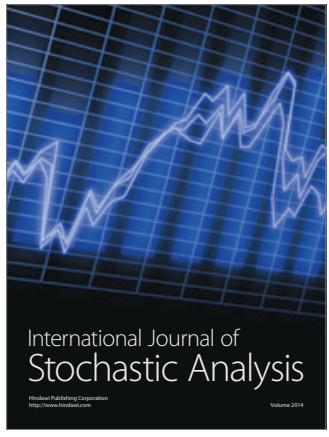

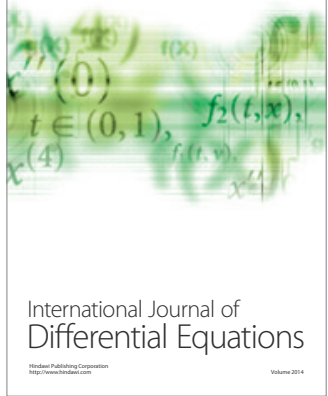
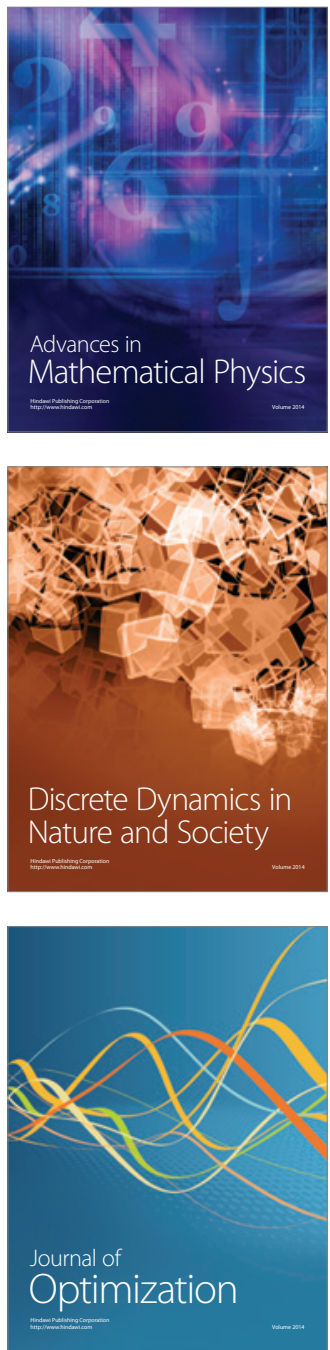